\title{
(6) OPEN ACCESS \\ Minimally invasive cone beam CT-guided evacuation of parenchymal and ventricular hemorrhage using the Apollo system: proof of concept in a cadaver model
}

\author{
David Fiorella, ${ }^{1}$ Adam Arthur, ${ }^{2}$ Sebastian Schafer ${ }^{3}$
}

- Additional material is published online only. To view please visit the journal online (http://dx.doi.org/10.1136/ neurintsurg-2014-011293)

${ }^{1}$ Department of Neurological Surgery, Stony Brook University Medical Center, Stony Brook, New York, USA

${ }^{2}$ Semmes-Murphey Clinic, Memphis, Tennessee, USA ${ }^{3}$ Siemens Medical Imaging, Erlangen, Germany

\section{Correspondence to} Dr D Fiorella, Department of Neurological Surgery, Stony Brook University Medical Center, Cerebrovascular Center, Health Sciences Center T-12 080, Stony Brook, NY 11794-8122, USA; david.fiorella@sbumed.org

Received 19 May 2014 Accepted 3 June 2014 Published Online First 1 July 2014

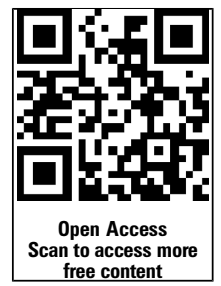

\section{ABSTRACT}

Introduction The Apollo system (Penumbra Inc, Alameda, California, USA) is a low profile irrigationaspiration system designed for the evacuation of intracranial hemorrhage.

Objective To demonstrate the feasibility of using Apollo in combination with cone beam CT guidance. Methods Parenchymal $(n=1)$ and mixed parenchymalintraventricular hematomas $(n=1)$ were created in cadaver heads using a transvascular $(n=1)$ or transcranial $(n=1)$ approach. Hematomas were then imaged with cone beam CT (CB-CT), and the long axis of the hematoma defined. The $\mathrm{CB}-\mathrm{CT}$ data were then used to guide transcranial access to the hematoma-defining the location of the burr hole and the path to the leading edge of the hematoma. An $8 \mathrm{~F}$ vascular sheath was then placed under live fluoroscopic guidance into the hematoma. A second CB-CT was performed to confirm localization of the sheath. The hematoma was then demarcated on the CB-CT and the Apollo wand was introduced through the $8 \mathrm{~F}$ sheath and irrigationaspiration was performed under (periodic) live fluoroscopic guidance. The operators manipulated the wand within the visible boundaries of the hematoma. After irrigation-aspiration, a control CB-CT was performed to document reduction in hematoma volume. Results Transvascular and transcranial techniques were both successful in creating intracranial hematomas. Hematomas could be defined with conspicuity sufficient for localization and volumetric measurement using CB-CT. Live fluoroscopic guidance was effective in navigating a sheath into the leading aspect of a parenchymal hematoma and guiding irrigation-aspiration with the Apollo system. Irrigation-aspiration reduced the parenchymal hemorrhage volume from 14.8 to $1.7 \mathrm{cc}$ in $189 \mathrm{~s}$ in the first case (parenchymal hemorrhage) and from 26.4 to $4.1 \mathrm{cc}$ in $300 \mathrm{~s}$ in the second case (parenchymal and intraventricular hemorrhage). Conclusions The cadaver model described is a useful means of studying interventional techniques for intracranial hemorrhage. It seems feasible to use CB-CT to guide the evacuation of intraparenchymal and intraventricular hemorrhage using the Apollo system through a minimally invasive transcranial access.

\section{INTRODUCTION}

The Apollo is a new endoscopically guided irrigation-aspiration system recently cleared by the US
Food and Drug Administration for the evacuation of intraventricular hemorrhage (figure 1).

Cone beam CT (CB-CT; DynaCT, Siemens Healthcare, Forchheim, Germany) has been shown to have adequate contrast resolution to differentiate intracranial hemorrhage from brain parenchyma and cerebrospinal fluid, and sufficient contrast and spatial resolution to allow the measurement of intracranial hemorrhage. ${ }^{1-3}$ CB-CT has also been shown to be a useful tool to guide transcranial ventricular access. ${ }^{4}$

The application of CB-CT to guide hematoma evacuation potentially provides the significant advantage of a much smaller cranial access ( $8 \mathrm{~F}$ vs 14-22F) than if the procedure was performed under endoscopic guidance as well as the opportunity for the seamless, periodic, real-time, intraprocedural evaluation of hematoma volume and morphology.

We present the first assessment of the feasibility of CB-CT-guided hematoma evacuation using the Apollo system in a cadaver model.

\section{METHODS}

\section{Cadaver models}

Two cadaver heads were used in this experiment. Both were immobilized in a radiolucent head holder on the angiography table (DORO, PMI, Freiburg, Germany). All imaging was performed with an Artis Zeego (Siemens Healthcare, Forchheim, Germany) angiographic system.

\section{Transvascular hematoma creation}

In the first cadaver, a standard $6 \mathrm{~F}$ vascular sheath (figure 2A) was inserted into the internal carotid artery and sutured into position. An aspiration catheter (5MAX Penumbra Inc, Alameda, California, USA) was then manipulated to the level of the carotid terminus under fluoroscopic roadmap control. A microcatheter $(027$ High Flo Renegade, Boston Scientific, Fremont, California, USA) was then introduced and manipulated to the distal tip of the aspiration catheter. The back end of a standard 0.014" microwire was then loaded into the microcatheter and used to perforate the carotid terminus. The microcatheter and then the aspiration catheter were advanced over the microwire into the basal ganglia (figure 2B). Cone beam CT was then performed to localize the tip of the aspiration catheter within the brain parenchyma 


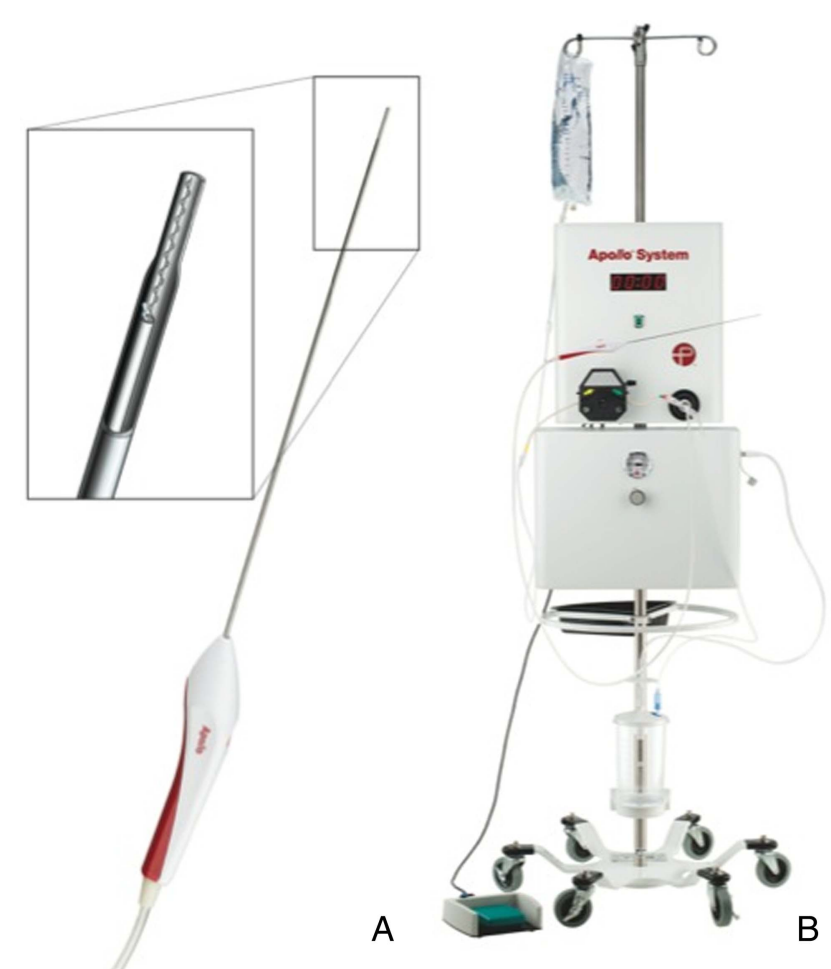

Figure 1 The Apollo system is composed of an aspiration-irrigation system, which can be attached via flexible tubing to the Apollo wand. The wand (A) can be accommodated by an $8 \mathrm{~F}$ vascular sheath.

The $2.6 \mathrm{~mm}$ wand houses an internal agitator wire that macerates clot material to maintain patency of the system during aspiration. The wand is attached to the freestanding aspiration-irrigation system (B). The aspiration-irrigation system (B) provides the capability for aspiration and saline irrigation and transmits vibrational energy to the internal agitator element within the wand.

(figure 2C). Next, coagulated pig blood was injected through the aspiration catheter directly into the brain parenchyma. The coagulated pig blood was a mixture of blood clot, noncoagulated serum, and saline. The catheter was removed, and CB-CT was repeated to document the size and configuration of the hemorrhage (figure 2D).

\section{Transcranial hematoma creation}

In the second cadaver, a burr hole was created at the midpupillary line, about $10 \mathrm{~cm}$ posterior to the glabella, and the dura was incised. An $18 \mathrm{~F}$ sheath was advanced under fluoroscopic guidance into the periventricular white matter. The coagulated pig blood solution was then injected through the sheath, directly into the brain. CB-CT was repeated to define the volume, morphology, and location of the hematoma (figure $3 \mathrm{~A}$ ).

\section{CB-CT-guided hematoma evacuation}

After creation and documentation of the hematomas, CB-CT data were loaded into the CB-CT guidance software (iGuide, Siemens Healthcare, Forchheim, Germany). The long axis of the hematoma was defined and extrapolated to the cranial surface (figure 2E, F). This point on the cranial surface was demarcated and targeted for burr hole creation. A site on the surface of the hematoma was also demarcated as the target for sheath placement. These two points were used to define the site of burr hole creation and the trajectory for sheath insertion. The distance between the cranial surface and the leading edge of the hemorrhage was measured. The robotic C-arm system was manipulated into a 'down-the-barrel' configuration, the target trajectory extending orthogonal to the center of the imaging plane. The site for the burr hole drilling was demarcated physically using the system's laser crosshair and simultaneously displayed as an overlay on live fluoroscopy (see figure $2 \mathrm{G}$, online supplementary movie 1 ). A standard $8 \mathrm{~F}$ vascular sheath was cut to match the length of the distance between the scalp surface and the leading edge of the hematoma and then manipulated under live fluoroscopic guidance along the path defined by CB-CT (see online supplementary movie 1). Once in position, repeat CB-CT was performed to confirm the location of the sheath and its orientation with respect to the configuration of the hemorrhage (figure $2 \mathrm{H}$ ).

The CB-CT data were then post-processed into three multiplanar reformations. The boundaries of the hematomas were manually demarcated and used to define a three-dimensional target for live fluoroscopic guidance (iGuide, Siemens Healthcare, Forchheim, Germany) of the Apollo system. The irrigation-aspiration wand was then introduced into the sheath under live fluoroscopic guidance. After confirmation that the tip of the wand was within the targeted volume from several different robotic C-arm positions, the irrigation-aspiration system was activated. Hematoma material could be visualized within the aspiration tubing as it was evacuated from the cavity. The stability of the position of the probe was occasionally checked with fluoroscopy. When the aspiration tubing contained only clear aspirate, the position of the wand was manipulated through the sheath into a different location within the demarcated hematoma cavity under live fluoroscopic guidance and aspiration was repeated. After aspiration, CB-CT was performed to assess the size and configuration of the remaining hematoma (figures $2 \mathrm{I}, \mathrm{J}$ and $3 \mathrm{~B}, \mathrm{C}$ ).

\section{Measurements}

Hematoma volumes were measured and manually demarcated on consecutive axial multiplanar re-formations with $1 \mathrm{~mm}$ slice thickness. $^{6}$ In addition, a second volume calculation was performed using the maximal orthogonal dimensions of the (ellipsoid) hematomas $(\mathrm{V}=(4 / 3) \pi \cdot \mathrm{a} \cdot \mathrm{b} \cdot \mathrm{c})$.

\section{RESULTS}

\section{Cadaver models}

Intracranial hemorrhages could be successfully created using either a transvascular or transcranial approach. The transvascular approach through the carotid terminus created a prototypical 'hypertensive-type' hemorrhage within the deep gray matter nucleus (figure 2D). The transvascular injection of hematoma material into the brain parenchyma through the aspiration catheter was met with some resistance and hematoma material was mixed with additional saline to facilitate injection. Injection via the transcranial approach through an $18 \mathrm{~F}$ access was considerably easier, but created a periventricular parenchymal hemorrhage, which decompressed into the ventricular system (figure 3A, B).

\section{Cone beam CT}

The injected blood products created a hematoma, with a density that could be easily differentiated from that of the adjacent brain parenchyma (figures 2D and 3A, B). This allowed measurement of the volume of the hemorrhage and demarcation of the hemorrhage for CB-CT-guided evacuation.

\section{CB-CT-guided Apollo procedure}

CB-CT facilitated the accurate placement of the 8F guiding sheath into the hematoma with the tip positioned in the superficial aspect of the hematoma and the sheath oriented parallel to 

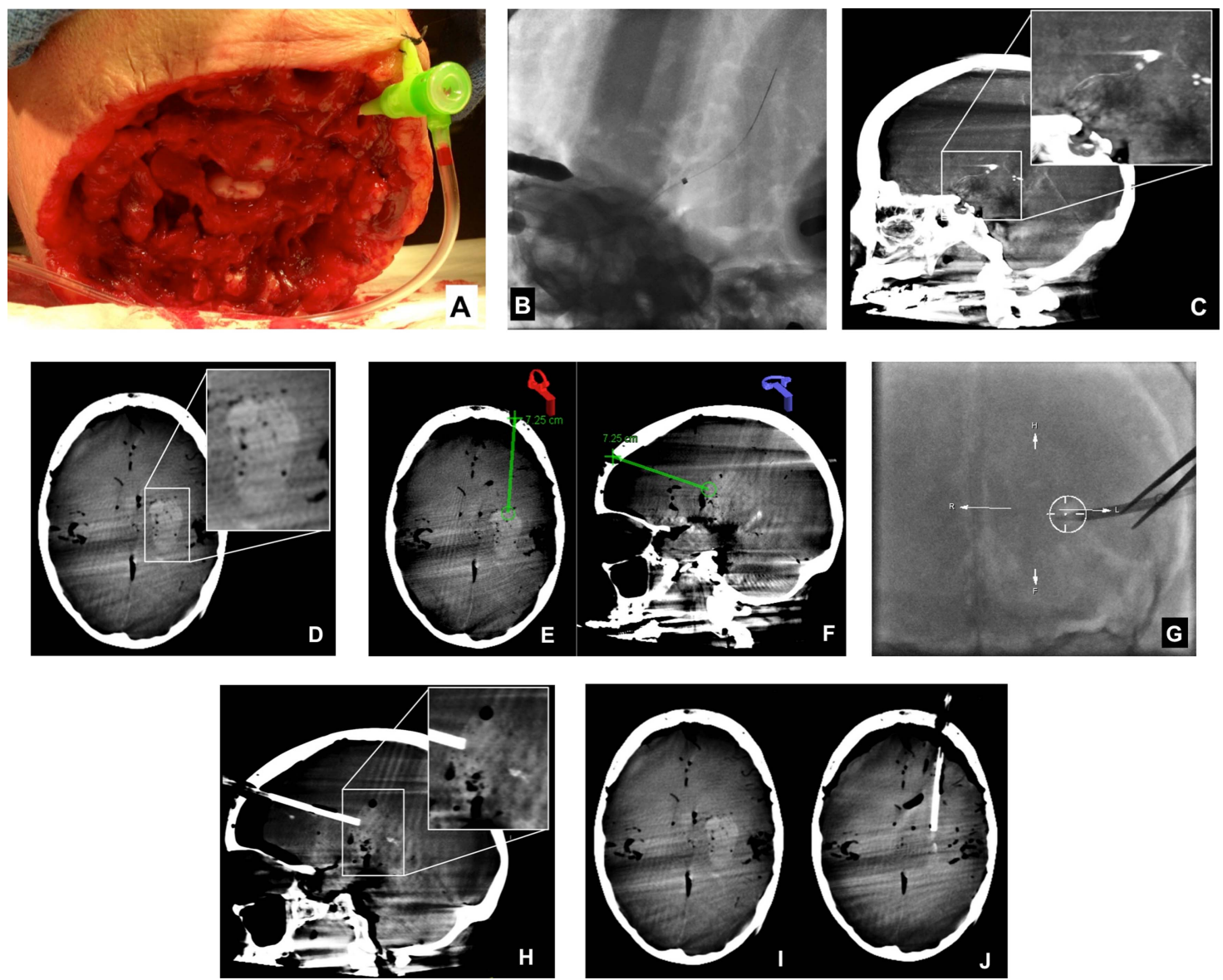

Figure 2 Transvascular hematoma creation and CB-CT-guided hematoma evacuation with the Apollo system. A standard 6F sheath was inserted directly into the left internal carotid artery (A) and sutured into position. Next, a 5MAX catheter with an internal Hi Flo Renegade microcatheter was positioned at the left carotid terminus. After perforation of the carotid terminus with a microwire, the 5MAX catheter was manipulated over the Renegade microcatheter and microwire into the brain parenchyma (B). A sagittal reformation of cone beam CT data performed to document the position of the catheter tip (C), demonstrated its placement within the ipsilateral basal ganglia. After infusion of blood products through the $5 \mathrm{MAX}$ catheter and removal of the catheter, CB-CT confirmed the successful creation of a parenchymal hematoma (D). Using points demarcated on CB-CT and registered using the iGuide software (E and F), an $8 \mathrm{~F}$ sheath was manipulated under live fluoroscopic guidance into the superficial aspect of the hematoma along its long axis. CB-CT was repeated to document the location of the sheath, demonstrating its position within the leading edge of the hematoma, in line with the long axis of the clot $(\mathrm{H})$. These $\mathrm{CB}-\mathrm{CT}$ data were then registered using the iGuide software, and the Apollo wand was introduced through the sheath freehand and activated to achieve clot evacuation under fluoroscopic guidance (see online supplementary movie 2). After clot evacuation, repeat CB-CT showed a marked reduction in the volume of hematoma with resolution of the local mass effect (l, J).

the long axis of the hematoma in both cases (figure $2 \mathrm{H}$ ). CB-CT enabled real-time fluoroscopic-guided evacuation of the hemorrhages using the Apollo system (figure 2I, J, 3B, C).
Ultimately the hematoma volumes (manually demarcated areas on consecutive axial multiplanar reconstruction images) were reduced from 14.8 to $1.7 \mathrm{cc}(88.5 \%)$ with $189 \mathrm{~s}$ of
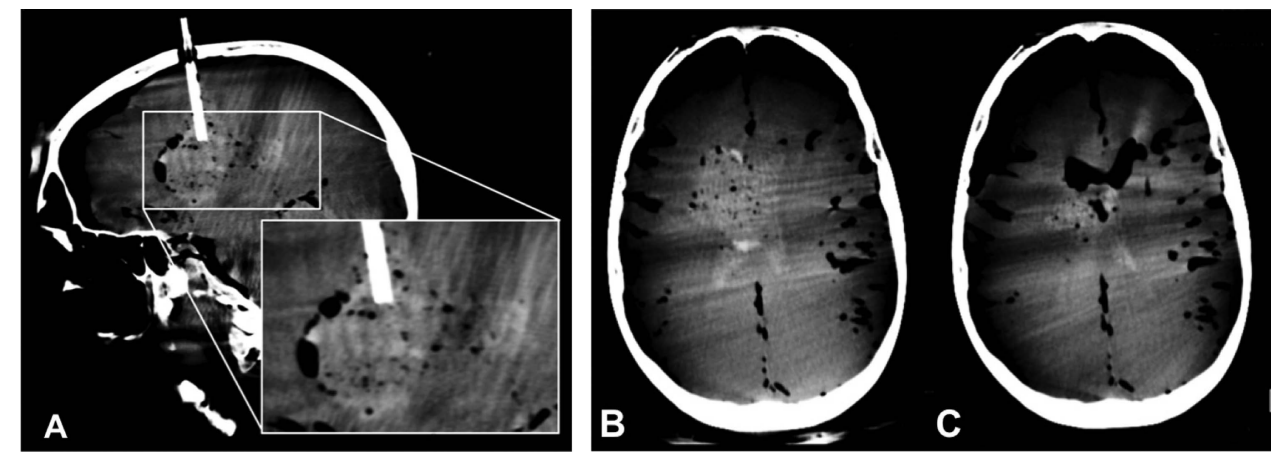

Figure 3 Transcranial hematoma creation and CB-CT-guided hematoma evacuation with the Apollo system. Sagittal re-formation of cone beam CT data confirms placement of an $18 \mathrm{~F}$ sheath extending into the right periventricular white matter via a right coronal burr hole with successful creation of a right parenchymal hematoma which has ruptured into the adjacent right lateral ventricle (A). Axial re-formation of CB-CT data before (B) and after (C) the evacuation of hemorrhage with the Apollo system shows a marked reduction in parenchymal and intraventricular blood products. The parenchymal hemorrhage cavity has now collapsed and the ventricular system has been decompressed and now contains air rather than blood. 
irrigation-aspiration in the first case (parenchymal hemorrhage) and from 26.4 to $4.1 \mathrm{cc}(84.2 \%)$ in $300 \mathrm{~s}$ in the second case (parenchymal and intraventricular hemorrhage). The total volumes of aspirated material (saline and blood products) measured in the collection canisters were 110 and $190 \mathrm{cc}$ for cases 1 and 2 . When the maximal dimensions of the hematoma were used to calculate hemorrhage volumes, the first hemorrhage measured $14.2 \mathrm{cc}$ and was reduced to a volume of $1.6 \mathrm{cc}$ and the second measured 25.2 and was reduced to a volume of $5.5 \mathrm{cc}$. Manual demarcation of the hematoma volume remaining after evacuation was more precise than the typical long-axis volumetric calculations, given the more dispersed configuration of the residual blood products.

\section{DISCUSSION AND CONCLUSIONS}

Intracranial hemorrhage (ICH) is a devastating disease, substantially more common than subarachnoid hemorrhage, accounting for between $10 \%$ and $15 \%$ of all strokes. ${ }^{7}$ Up to half of patients with ICH do not survive their initial hospitalization and an additional one-third require long-term care after discharge. ${ }^{8}$ No medical or open surgical interventions improve outcomes. ${ }^{9-12}$

Many reasons have been proposed to explain the failure of open surgical treatments to improve outcomes in patients with ICH. One of the more common explanations is that regional trauma caused to uninjured brain by the open surgical resection offsets any benefit offered by the surgical evacuation. ${ }^{9}$ More recently the Minimally Invasive Surgery and tPA in ICH evacuation (MISTIE II) trial provided evidence that minimally invasive hematoma evacuation could reduce peri-hematoma edema, improve patient outcomes, reduce length of hospital stay, and reduce mortality. ${ }^{13}{ }^{14}$ Moreover, the investigators observed that greater procedural reductions in clot volume correlated with better patient outcomes. Minimally invasive hematoma decompression is theorized to be effective by reducing the direct toxic effect of blood products on the surrounding brain parenchyma and by alleviating local mass effect, which can create regional ischemia in the surrounding parenchyma. ${ }^{15} 16$

The MISTIE procedure involves manual syringe aspiration of a hematoma with a $14 \mathrm{~F}$ cannula, followed by placement of a smaller bore drainage catheter, which can be irrigated with tissue plasminogen activator to maintain its patency. ${ }^{13}$ The Apollo system potentially enables more thorough initial clot evacuation through a much lower profile system $(<8 \mathrm{~F})$. The patency of the system is maintained by an internal agitator wire within the aspiration wand, which vibrates and macerates hematoma, preventing the apparatus from becoming clogged with blood products despite its low profile. For this reason, if the wand can be introduced into a hematoma cavity through a guiding sheath, it is possible that the goal reduction in hematoma volume $(80-90 \%)$ can be achieved efficiently with the initial interventional procedure, particularly if the status of the hematoma removal can be monitored.

As cone beam CT systems have evolved, integrated point-to-point and volume-based live fluoroscopic navigation software packages have been developed. These software packages allow the real-time superimposition of data derived from CB-CT imaging onto a live, two-dimensional, fluoroscopic image. This enables a myriad of imaging-guided interventional procedures within the angiography suite (eg, percutaneous spine interventions, complex peripheral vascular interventions). ${ }^{17-20}$ Recently, use of the CB-CT-guided technique has been reported for the placement of external ventricular drains within the angiography suite. ${ }^{4}$
Hematoma evacuation under CB-CT guidance has several potential advantages. First, the procedure can be performed through a small transcranial access $(8 \mathrm{~F})$, minimizing trauma to the brain and potentially maximizing the beneficial effect of the procedure. Second, performing the procedure on an angiographic unit with CB-CT capability, allows immediate CT assessment of the status of the hematoma and brain without having to transfer the patient. CT can indicate the need for further evacuation, direct the operator to a different component of the hematoma, and promptly identify any complications of the procedure. CT is the standard imaging modality used to assess intracerebral hemorrhage as it allows visualization of the entire extent of the hemorrhage as well as the surrounding brain with excellent contrast resolution. Thus, CB-CT is possibly to be a more accurate predictor of remaining hemorrhage volume than is direct visualization with an endoscope-which may provide a limited visualization of some components of a parenchymal hemorrhage. For these reasons, by allowing the operator to periodically assess the status of the hemorrhage evacuation, the CB-CT-guided technique has the potential to facilitate a more complete initial hematoma evacuation. At the same time, there are some potential disadvantages of a sole CB-CT guided approach. First, it is impossible to visualize what is happening at the tip of the Apollo Wand if no blood products are aspirating from the cavity. Second, in the event of re-bleeding, there is limited opportunity to achieve hemostasis with targeted irrigation or cautery as there potentially is with endoscopy. Ultimately some combination of the two approaches-CB-CT with endoscopy may prove to be the optimal approach to this procedure. However, this remains to be determined.

The present cadaver head intracranial hemorrhage model provides a straightforward means of creating intracranial hematomas. The transvascular approach created a basal ganglia hemorrhage, resembling a typical hypertensive hemorrhage. The transcranial approach created a periventricular hemorrhage, which ruptured into the lateral ventricle. The cadaver model is optimal for the evaluation of devices, as the sizes of the cranial compartment and brain in subhuman species are much smaller and considerably more difficult to work with.

Using this model, it was feasible to use the Apollo system to evacuate hemorrhages under CB-CT guidance alone. CB-CT facilitated the placement of a vascular sheath into the leading edge of the experimental hematoma. Live fluoroscopic guidance was sufficient to direct the free-hand insertion of the Apollo probe into the hematoma cavity and guide its manipulation within the hematoma cavity. Post-Apollo CB-CT demonstrated the remaining hematoma with excellent conspicuity. The Apollo system allowed the efficient removal of the majority $(>80 \%)$ of the hematoma volume within a very short period to time $(3-5 \mathrm{~min})$.

In summary, the cadaver model of intracranial hemorrhage described is useful for the evaluation of devices designed for the minimally invasive evacuation of parenchymal and/or intraventricular hemorrhages. It is feasible to use CB-CT to guide the transcranial placement of sheaths and devices designed for minimally invasive hematoma evacuation. The Apollo system allowed the efficient and almost total evacuation of a parenchymal and mixed parenchymal-intraventricular hemorrhage through a minimally invasive access in these cadaver models.

Contributors All authors made substantial contributions to the conception and design, acquisition of data, or analysis and interpretation of data.

Funding State University of NY at Stony Brook receives research grants from Siemens Medical Imaging. This study was supported in part by a grant from Siemens 
Medical Imaging and Penumbra Inc. The Apollo program at SBUMC is also supported by a grant from the Stony Brook University Hospital Auxiliary.

Competing interests SS is an employee of Siemens Medical Imaging.

Provenance and peer review Not commissioned; externally peer reviewed.

Open Access This is an Open Access article distributed in accordance with the Creative Commons Attribution Non Commercial (CC BY-NC 4.0) license, which permits others to distribute, remix, adapt, build upon this work non-commercially, and license their derivative works on different terms, provided the original work is properly cited and the use is non-commercial. See: http://creativecommons.org/ licenses/by-nc/4.0/

\section{REFERENCES}

1 Struffert T, Deuerling-Zheng Y, Kloska S, et al. Flat detector $\mathrm{CT}$ in the evaluation of brain parenchyma, intracranial vasculature, and cerebral blood volume: a pilot study in patients with acute symptoms of cerebral ischemia. AJNR Am J Neuroradiol 2010;31:1462-9.

2 Struffert T, Eyupoglu IY, Huttner HB, et al. Clinical evaluation of flat-panel detector compared with multislice computed tomography in 65 patients with acute intracranial hemorrhage: initial results. Clinical article. J Neurosurg 2010;113:901-7.

3 Rouchaud A, Pistocchi S, Blanc R, et al. Predictive value of flat-panel CT for haemorrhagic transformations in patients with acute stroke treated with thrombectomy. J Neurointervent Surg 2014;6:139-43.

4 Fiorella D, Peeling L, Denice CM, et al. Integrated flat detector CT and live fluoroscopic-guided external ventricular drain placement within the neuroangiography suite. J Neurointervent Surg 2014;6:457-60.

5 Cooke DL, Levitt M, Kim LJ, et al. Transcranial access using fluoroscopic flat panel detector CT navigation. AJNR Am J Neuroradiol 2011;32:E69-70.

6 Yushkevich PA, Piven J, Hazlett HC, et al. User-guided 3D active contour segmentation of anatomical structures: significantly improved efficiency and reliability. Neuroimage 2006;31:1116-28.

7 Qureshi Al, Mendelow AD, Hanley DF. Intracerebral haemorrhage. Lancet 2009;373:1632-44.
8 Russell MW, Boulanger L, Joshi AV, et al. The economic burden of intracerebral hemorrhage: evidence from managed care. Manag Care Interface 2006;19: $24-8,34$.

9 Abdu E, Hanley DF, Newell DW. Minimally invasive treatment for intracerebral hemorrhage. Neurosurg Focus 2012;32:E3.

10 Andrews CM, Jauch EC, Hemphill JC III, et al. Emergency neurological life support: intracerebral hemorrhage. Neurocrit Care 2012;(17 Suppl 1):S37-46.

11 Sacco S, Marini C, Toni D, et al. Incidence and 10-year survival of intracerebral hemorrhage in a population-based registry. Stroke 2009;40:394-9.

12 Broderick JP, Brott TG, Duldner JE, et al. Volume of intracerebral hemorrhage. A powerful and easy-to-use predictor of 30-day mortality. Stroke 1993;24:987-93.

13 Mould WA, Carhuapoma JR, Muschelli J, et al. Minimally invasive surgery plus recombinant tissue-type plasminogen activator for intracerebral hemorrhage evacuation decreases perihematomal edema. Stroke 2013;44:627-34.

14 Hanley DF. 365-day outcome and cost model. Mistie II: a phase II proof-of-concept trial. International Stroke Conference. 2013.

15 Arima $\mathrm{H}$, Wang JG, Huang $Y$, et al. Significance of perihematomal edema in acute intracerebral hemorrhage: the interCB-CT trial. Neurology 2009;73:1963-8.

16 Gebel JM Jr, Jauch EC, Brott TG, et al. Natural history of perihematomal edema in patients with hyperacute spontaneous intracerebral hemorrhage. Stroke 2002;33:2631-5.

17 Freundt Ml, Ritter M, Al-Zghloul M, et al. Laser-guided cervical selective nerve root block with the dyna-CT: initial experience of three-dimensional puncture planning with an ex-vivo model. PloS ONE 2013;8:e69311.

18 Czerny C, Eichler $\mathrm{K}$, Croissant $Y$, et al. Combining C-arm CT with a new remote operated positioning and guidance system for guidance of minimally invasive spine interventions. J Neurointervent Surg 2015;7:303-8.

19 Dijkstra ML, Eagleton MJ, Greenberg RK, et al. Intraoperative C-arm cone-beam computed tomography in fenestrated/branched aortic endografting. I Vasc Surg 2011:53:583-90

20 van Bindsbergen L, Braak SJ, van Strijen MJ, et al. Type ii endoleak embolization after endovascular abdominal aortic aneurysm repair with use of real-time three-dimensional fluoroscopic needle guidance. I Vasc Interv Radiol 2010;21:1443-7. 